\title{
A hybrid passive localization method under strong interference with a preliminary experimental demonstration
}

\author{
Bo Lei", Yixin Yang, Kunde Yang, Yong Wang and Yang Shi
}

\begin{abstract}
Strong interference exists in many passive localization problems and may lead to the inefficacy of traditional localization methods. In this study, a hybrid passive localization method is proposed to address strong interference. This method combines generalized cross-correlation and interference cancellation for time-difference-of-arrival (TDOA) measurement, followed by a time-delay-based iterative localization method. The proposed method is applied to a preliminary experiment using three hydrophones. The TDOAs estimated by the proposed method are compared with those obtained by the particle filtering method. Results show that the positions are in agreement when the TDOAs are accurately obtained. Furthermore, the proposed method is more capable of localization in the presence of a strong moving jamming source.
\end{abstract}

Keywords: Interference cancellation, Radon transform, Cross-correlation, Localization, Underwater acoustics

\section{Introduction}

Passive source localization is a significant and important topic in signal processing because of its minimal impact on the environment and low susceptibility to the effects of clutter. This viable approach has certain advantages in navigation [1], speaker tracking [2], radar [3], and underwater acoustics $[4,5]$. The time-delay-based method is the most widely used localization strategy, which is a two-step scheme. In general, time-difference-of-arrival (TDOA) measurements of a passive signal on spatially separate receivers are first estimated, followed by the solution of nonlinear hyperbolic equations using the range-difference information obtained from the product of the measured time delays and the known propagation speed. Thus, the source position can then be determined based on the sensor array geometry.

In localization, a straightforward TDOA estimation between a pair of receivers can be realized by determining the peak of the cross-correlation function. A generalized cross-correlation called the phase transform (PHAT) [6], which uses the normalized spectra of the signals, is commonly used in time-delay measurements [7-9]. The

\footnotetext{
* Correspondence: lei.bo@nwpu.edu.cn

School of Marine Science and Technology, Northwestern Polytechnical University, Xi'an 710072, China
}

position of the source can be estimated through the intersection point of each pair of hyperbolic functions. However, since each pair can have zero, one, or two intersections, the logic to find the correct one is nontrivial. Also, determining the correct weighting is difficult. Solving the hyperbolic functions using nonlinear least squares has been considered as a possible approach [10], in which a Taylor-series expansion is used for linearization and the solution is determined iteratively. A two-step weighted leastsquares algorithm proposed by Chan [11] could provide the final solution of the position coordinates by exploiting the known relation between the intermediate variable and the position coordinates. Young et al. [12] explored the use of cross-correlation-based TDOA methods for localization by a modified minimum-variance distortionless response technique. Lui [13] derived a semi-definite programming algorithm for source localization by integrating some available prior information. Friedlander [14] estimated the range and depth of an underwater source by measuring the propagation delay differences among multiple propagation paths on two vertically deployed receivers. Felisberto et al. [15] further developed a localization method that minimizes a time-delay objective function with respect to the depth and range with the use of a 
ray-based backpropagation algorithm. Particle filtering (PF) has also been used to automate detection and localization $[16,17]$.

Furthermore, a number of approaches have been proposed for multitarget localization. A PF-based algorithm was developed for the localization and tracking of multiple acoustic sources in reverberative environments [18]. Based on maximum-likelihood estimation, a technique using two omnidirectional passive sensors for the detection and estimation of a target in the presence of false measurements was developed [19], where the target motion parameters are obtained by directly maximizing a joint-likelihood function. A multitarget tracking formulation [20] was studied as an incomplete data problem, where a maximum-likelihood estimator was derived based on an expectation-maximization algorithm [21]. The likelihood estimator is maximized indirectly by iterating the expectation and maximization steps until certain appropriate convergence conditions are satisfied and is successfully applied to the state estimation of nonmaneuvering targets in a cluttered underwater environment. A nonlinear least-square technique was used to compute the motion parameters for each target by modeling the Gaussian mixture probability density functions of TDOA measurement errors [22].

Without loss of generality, the localization technique described in this paper is based on TDOA measurement between spatially separated hydrophones. However, a problem originates from the boat-noise target signal being totally polluted by strong interference. This contamination is common in a coastal environment and may be in the form of interference from moving merchant ships, artificial noise, and marine mammal bioacoustics, among others. If the signal-to-interference ratio is low, then localization may be inaccurate when the TDOAs of the two signals are close. Therefore, the interference has to be canceled before TDOA estimation. Accordingly, this paper presents a hybrid localization method integrated with interference cancellation. The method comprises three steps. First, PHAT processing is applied to the recorded data. Second, an interference cancellation method involving the Radon transform $[23,24]$ is exploited, so that the TDOAs on each pair of receivers are accurately acquired. Finally, iteration is performed to search for the source position. The proposed method is validated by a preliminary experiment, in which a moving jamming source is present.

The remainder of the paper is organized as follows: Section 2 presents the framework of the hybrid localization method. In Section 3, an experimental demonstration with three spatially separated hydrophones in a lake is provided. Section 4 further discusses the proposed method, with a moving interference taken into consideration. Section 5 presents the conclusions of this study.

\section{Framework of the method}

In underwater localization, the target signals are contaminated by strong interference, and this contamination may result in inaccurate TDOA measurement. As a result, the efficacy of conventional localization methods is affected. The framework of the proposed localization method, which can cancel interference, comprises three processing steps, as shown in Fig. 1. PHAT processing is first applied to the received data from each pair of receivers, followed by a novel interference cancellation method involving the Radon transform, whereby, the TDOAs of the target signal are obtained. The final process is to localize the target source based on the estimated TDOAs of all receiver pairs.

\subsection{PHAT processing}

Let $s(t)$ and $p(t)$ represent the boat-noise target signal and interference from a jamming source, respectively, and $x_{1}(t)$ and $x_{2}(t)$ represent the signals received by two hydrophones located at distant locations and arranged in a known geometry. These two received signals are respectively expressed as

$$
\begin{aligned}
& x_{1}(t)=s\left(t-D_{1}\right)+p\left(t-D_{\mathrm{i} 1}\right)+n_{1}(t), \\
& x_{2}(t)=s\left(t-D_{2}\right)+p\left(t-D_{\mathrm{i} 2}\right)+n_{2}(t),
\end{aligned}
$$

where the unknown parameters $D_{1}$ and $D_{2}$ are the time delays of the target signal on the two hydrophones, $D_{\mathrm{i} 1}$ and $D_{\mathrm{i} 2}$ are the time delays of the interference, and $n_{1}$ and $n_{2}$ are additive noises on the two hydrophones. In general, the interference is uncorrelated with the target signal $s(t)$. Thus, TDOAs $D_{1}-D_{2}$ and $D_{\mathrm{i} 1}-D_{\mathrm{i} 2}$ may be derived as two peaks of the common cross-correlation function between the two hydrophone signals. However, if the interference-to-signal ratio is strong, the peak of the

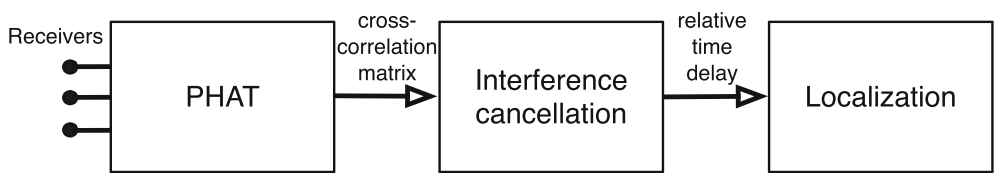

Fig. 1 Sketch of the proposed hybrid localization method, comprising three processing steps: PHAT processing, interference cancellation, and localization 
target signal output may be buried in the interference. The PHAT method, which is a generalized cross-correlation processing method, has the capability to suppress the interference power and can be mathematically expressed as

$$
y(t)=\operatorname{IFFT}\left(\frac{X_{1}(f) X_{2}^{*}(f)}{\left|X_{1}(f)\right|\left|X_{2}(f)\right|}\right)
$$

where * indicates complex conjugation and $X_{1}(f)$ and $X_{2}(f)$ are the spectra of $x_{1}(t)$ and $x_{2}(t)$, respectively. In the PHAT output $y(t)$, two peaks corresponding to the interference and target signal are present. When both TDOAs are close, the TDOA of the target signal is difficult to accurately obtain because the target-signal peak of the PHAT output is significantly obscured. Therefore, the interference should be suppressed before TDOA estimation. Even in cases in which the two peaks are totally separated, the cancellation process is beneficial to automatically determine the TDOA.

\subsection{Interference cancellation}

If a strong jamming source exists in the background, an obvious additional peak will appear in the PHAT output. In block processing, the sampled waveform of the target signal when the source is moving is divided into blocks, and PHAT processing is applied to each of the blocks. Once the PHAT outputs are organized into a crosscorrelation matrix, in which each row represents a PHAT output, a false trajectory corresponding to the peaks may be present along the running time dimension. In most cases, the trajectory does not exhibit a straight-line behavior. If the PHAT outputs are rearranged to generate a line for the dominant interference component, the Radon transform can be exploited, which is effective for line detection. On the basis of this intuition, a novel processing method is proposed for interference cancellation on the PHAT outputs. The procedure of this method is illustrated in Fig. 2 and described as follows:
1. Successive blocks of the received signals on each receiver pair are processed using the PHAT technique to generate a cross-correlation matrix. Given that the Radon transform renders good line detection, all the peaks of the PHAT outputs, which correspond to the dominant interference component in the matrix, are aligned to generate a line along the running time axis. Thus, a new matrix is generated, as shown on the left of Fig. 2. In this way, an output matrix $P$ with dimension $N \times M$ is produced, where $N$ is the number of processed blocks and $M$ is the length of the PHAT output. The output matrix has a nearly straight vertical line along the running time axis, and this line corresponds to peaks of the interference cross-correlation. The offset of each PHAT peak in the processing procedure is stored in memory for later use.

2. The first $M$ rows of $P$ are selected and form the block named $P_{1}$, which is of dimension $M \times M$ and, in this example, covers an event when the target signal and interference have the same or similar TDOAs. The second block following $P_{1}$, named $P_{2}$, is also of dimension $M \times M$. The Radon transform is performed on both matrices:

$$
\begin{aligned}
& P_{1 \mathrm{R}}=\operatorname{RT}\left(P_{1}\right), \\
& P_{2 \mathrm{R}}=\operatorname{RT}\left(P_{2}\right),
\end{aligned}
$$

where RT (.) denotes the Radon transform. The transformed matrix $P_{1 \mathrm{R}}$ contains both the TDOA variations of the interference and target signal along the running time dimension, whereas the matrix $P_{2 R}$ contains only information of the interference. If the target signal is partially contained in $P_{2}$, then a negative peak will appear in the interference cancellation result.

3. Let $\Delta P_{\mathrm{R}}=P_{1 \mathrm{R}}-P_{2 \mathrm{R}}$, so that the interference in $P_{1 \mathrm{R}}$ is canceled. The inverse Radon transform (IRT) is then applied to $\Delta P_{R}$, yielding

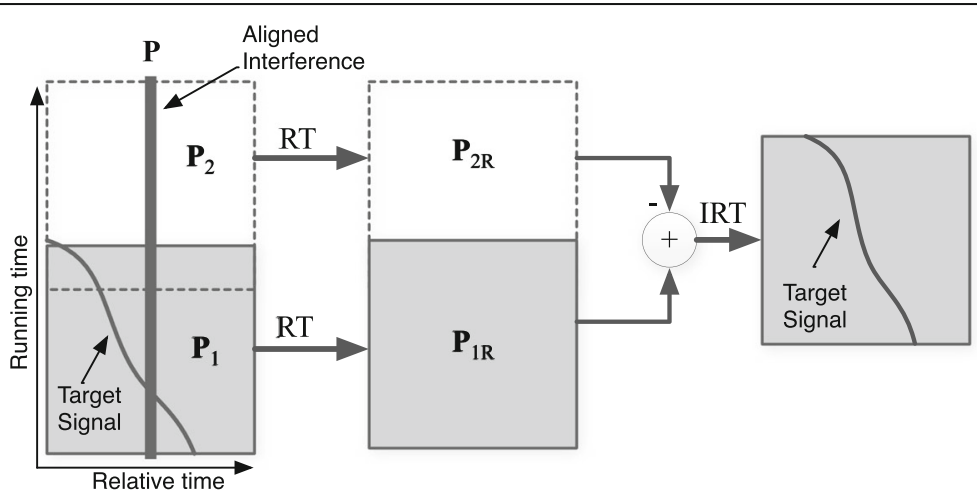

Fig. 2 Interference cancellation method, whereby the Radon transform is executed on two aligned matrices selected from the PHAT outputs, and the interference is canceled by the inverse Radon transform on their difference 


$$
\tilde{P}=\operatorname{IRT}\left(\Delta P_{\mathrm{R}}\right),
$$

As shown in (4), the PHAT output of the target signal is retained, whereas that of the interference is canceled. The TDOA of the target signal can then be evaluated according to the peak position on the relative time axis by undoing the recorded offset compensation from step 1 above.

Theoretically, parameter $M$ is independent of the moving speed of the source. Only variations in the peak values of the PHAT results can degrade the jamming signal cancellation performance. If the variation in the jamming signal is weak, then a large $M$ value may be set, and vice versa.

Once the TDOAs on the receiver pairs are determined, the position of the object is further assessed by estimating the intersection point of each pair of hyperbolic functions or determining the true position values by some other method.

\subsection{Localization algorithm}

$N$ receivers are assumed to be located at position $\left(a_{i}, b_{i}\right)$, and the general formulation of TDOA distance between Receiver $i$ and Receiver $j$ for source position $(X, Y)$ is mathematically described as

$$
\Delta d_{i j}=f\left(X, Y ; a_{i}, b_{i}, a_{j}, b_{j}\right), \quad 1 \leq i<j \leq N .
$$

The distance function $f$ can be estimated using the geometry for the direct wave without boundary interaction or using a ray model for multipath propagation. The nonlinear Eq. (5) is overdetermined when $N>3$, and the solution can then be derived by nonlinear least-squares estimation of $(X, Y)$, given by

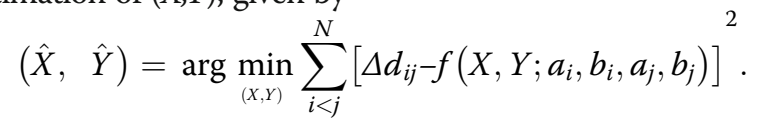

For simplification, $Q=(X, Y)$ is used to represent the position of the object. Therefore, with the use of a leastsquares criterion, the minimization problem in vector notation can be written as

$$
\hat{Q}=\arg \min _{\mathbf{Q}}\left\{[\Delta d-f(Q)]^{T} R^{-1}[\Delta d-f(Q)]\right\},
$$

where $\Delta d=\left(\Delta d_{1,2}, \cdots, \Delta d_{N-1, N}\right)^{T}$ and are the covariance matrix of the TDOA measurements. The minimumvariance solution can be obtained using the stochastic gradient algorithm

$$
Q^{m+1}=Q^{m}-\mu^{m} f_{\mathbf{Q}}^{\prime}\left(Q^{m}\right)\left[\Delta d-f\left(Q^{m}\right)\right],
$$

where $f_{Q}^{\prime}$ indicates the derivation of function $f$ with respect to $Q$. A good choice of step size is formulated by the normalization

$$
\mu^{m}=\frac{\mu}{\left\|f_{Q}^{\prime}\left(Q^{m}\right)\right\|^{2}} .
$$

The performance of the localization method depends on the accuracy of the TDOA measurement, $\Delta d$. For $N$ hydrophones, the maximum number of intersection points for each pair of hyperbolic functions is

$$
\left(\left(\begin{array}{c}
N \\
2 \\
2
\end{array}\right)\right)
$$

where $\left(\begin{array}{l}p \\ q\end{array}\right)=\frac{p !}{q !(p-q) !}$ is the number of combinations of $p$ things taken $q$ at a time.

Once the number of hydrophones exceeds 3 , the problem is overdetermined. Given time-delay errors caused by noise, waveguide fluctuation, and interference, a good solution may not be achieved if a large error exists on some of the hydrophones. Therefore, three hydrophones is a good choice for a practical localization system.

\section{Experimental demonstration}

\subsection{Experiment configuration}

A preliminary experiment was conducted in a lake with a depth of $40 \mathrm{~m}$, aimed to verify the localization method under strong interference with a limited number of hydrophones. The configuration is shown in Fig. 3. An omnidirectional broadband transmitter with center frequency of $10 \mathrm{kHz}$ was deployed as a jamming source at a depth of $10 \mathrm{~m}$ and range of $1100 \mathrm{~m}$. Owing to the limited conditions of this experiment, only three hydrophones were deployed at a depth of $10 \mathrm{~m}$, with the $\# 1$ and \#2 hydrophones being $5 \mathrm{~m}$ apart and \#2 and \#3 hydrophones being $10 \mathrm{~m}$ apart. The hydrophone outputs were followed by a pre-filter with a pass-band of $4-16 \mathrm{kHz}$ (to verify the proposed method under strong interference). A boat approximately $4 \mathrm{~m}$ in length was traveling at a speed of approximately $0.5 \mathrm{~m} / \mathrm{s}$, based on its global positioning system (GPS).

The sound speed profile measured by CTD (conductivity-temperature-depth) is shown in Fig. 4a. The upper isovelocity volume exhibits a constant sound speed of approximately $1484 \mathrm{~m} / \mathrm{s}$. In the lower volume, the sound speed profile shows a negative gradient because of the temperature decrease. The sound speed was measured down to a depth of $33 \mathrm{~m}$ because exact information below that level was not available. At the lower volume, the sound speed was evaluated according to the negative gradient with respect to a reference value of $1445 \mathrm{~m} / \mathrm{s}$ at a depth of $40 \mathrm{~m}$. The bottom was assumed to be a halfspace with a density of $1.6 \mathrm{~g} / \mathrm{cm}^{3}$ and a sound speed of $1720 \mathrm{~m} / \mathrm{s}$. This assumption did not bear any impact on the propagation path. 


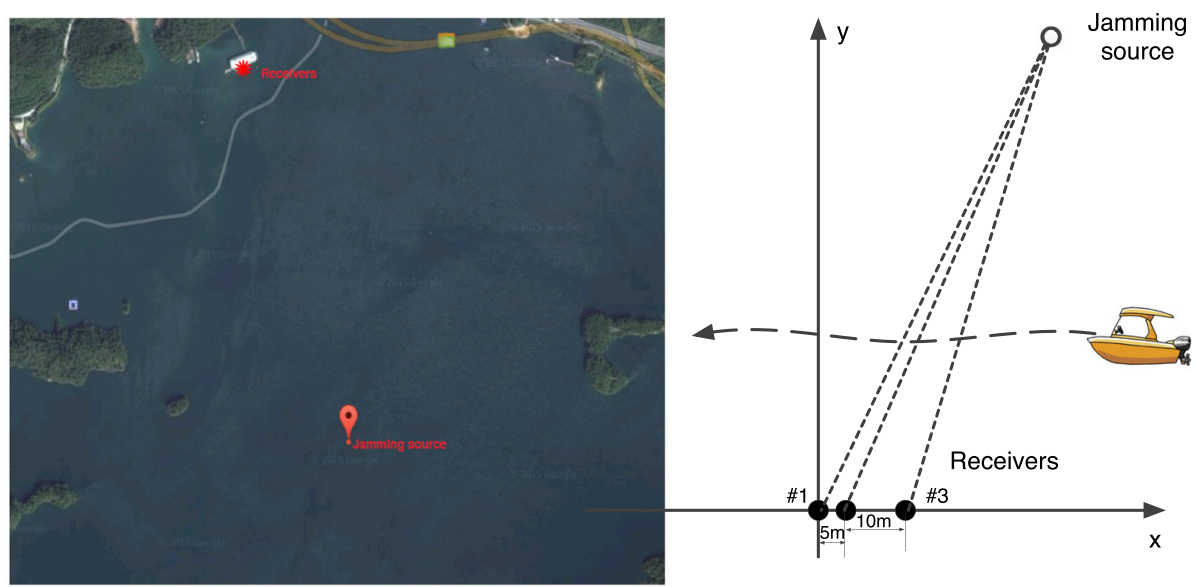

Fig. 3 Experimental configuration, showing three hydrophones at a depth of $10 \mathrm{~m}$, a boat traveling at low speed, and a jamming source at a distance of approximately $1100 \mathrm{~m}$ from the receivers

The rays propagated from the moving boat in this environment were computed using the Bellhop ray model $[25$, 26 ] with the source located at a depth of $0.5 \mathrm{~m}$, as shown in Fig. 4b. Most of the rays travel downward and are then reflected from the bottom. Direct-path waveforms were received at a depth of $10 \mathrm{~m}$ when the source range was less than $500 \mathrm{~m}$, and bounces at the boundaries occurred more than once when the source distance exceeded $700 \mathrm{~m}$.

As the boat moves beyond the receiver array, a $5-15 \mathrm{kHz}$ linear frequency modulation (LFM) signal was radiated from the jamming source with a duration of $0.1 \mathrm{~s}$ and repeated every $0.5 \mathrm{~s}$. Both the LFM signal and boat noise were simultaneously filtered and recorded. Even though the spectrum of the boat noise is lower at frequencies of only a few thousand hertz, it is more significant for the interference cancellation study. A portion of the waveform recorded on the \#1 hydrophone and its power spectrum are shown in Fig. 5. The plots show that the boat noise is approximately $25 \mathrm{~dB}$ lower than the LFM jamming signal and is therefore seriously contaminated.

Given that the jamming source was practically motionless, a line in the cross-correlation output matrix should exist. Therefore, the interference cancellation procedure can be simplified in subsequent processing because PHAT peak offsets are not necessary.

\subsection{Processing results and comparison}

All three hydrophone outputs were used to compute the TDOAs, as described in (5). Consequently, three hyperbolic functions were generated. The PHAT results from
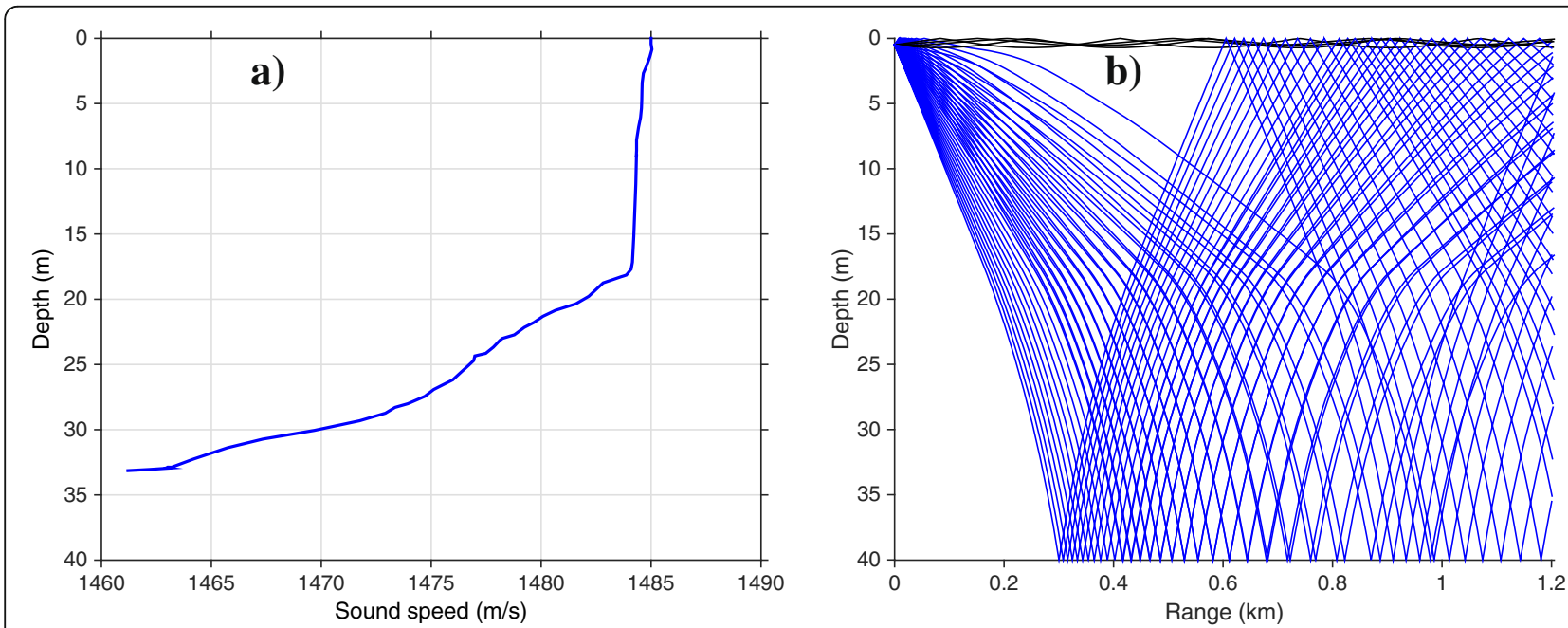

Fig. 4 Measured sound speed profile and propagation paths from the position of the small boat. a Sound speed, showing approximately constant value at depths of more than $18 \mathrm{~m}$ but a negative gradient below this depth. $\mathbf{b}$ Ray model outputs where the depth of the source (moving boat) was set to $0.5 \mathrm{~m}$ 

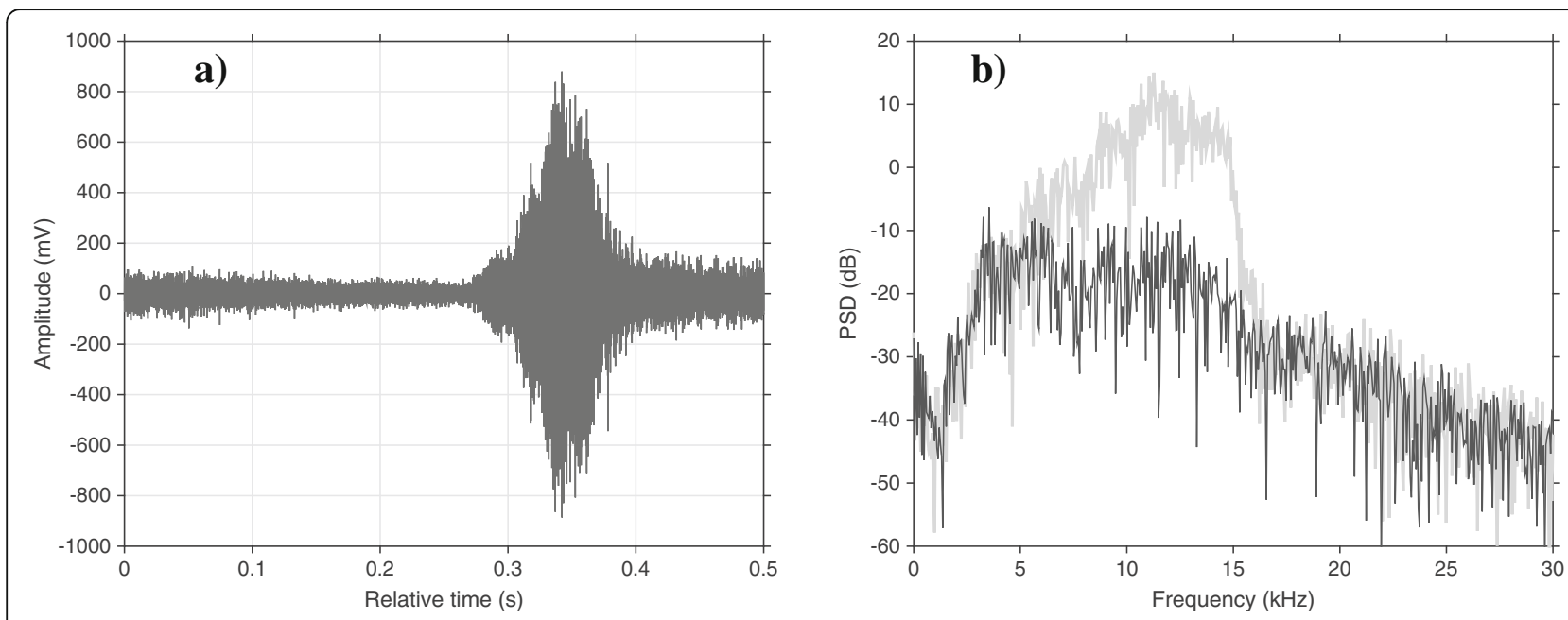

Fig. 5 Characteristics of signal received on \#1 hydrophone. a Received waveform. b Power spectral density, showing boat noise (dark line) significantly polluted by interference (light line)

the received data on the first pair of hydrophones (\#1 and \#2) are shown in Fig. 6a. The peaks of the crosscorrelation output of the boat noise are evident, owing to the spectral normalization of the interference by PHAT processing. The proposed interference cancellation method was then applied to the PHAT output, where parameter $M=400$ and pulses $1-400$ were selected for $P_{1}$, whereas pulses 11-410 were selected for $P_{2}$. The interference cancellation results in Fig. $6 \mathrm{~b}$ show that the interference was well suppressed throughout the entire running time, particularly at the crossing event. The TDOAs are corresponding to the time delays of the maximum values of the rows of matrix $\tilde{P}$ were finally determined, as shown in Fig. 7a. The result obtained by the PF method is shown in Fig. 7b for comparison. The PF method apparently tracked the wrong target at the crossing, whereas the proposed method provides a satisfactory assessment of the TDOAs.

The localization process was then performed using the assessed TDOA for each of the three receiver pairs. The results for the proposed method throughout the entire running time are shown in Fig. 7c, where $\mu=1$, showing that the boat traveled approximately along a straight line. By contrast, a portion of the results obtained using PF method is shown in Fig. 7d. Both methods have nearly the same localization results, with a difference not exceeding $20 \mathrm{~m}$ along the $y$ direction.

\section{Experiment on moving jamming source}

In Section 3, the jamming source is almost motionless and cooperative. In actual multitarget localization,
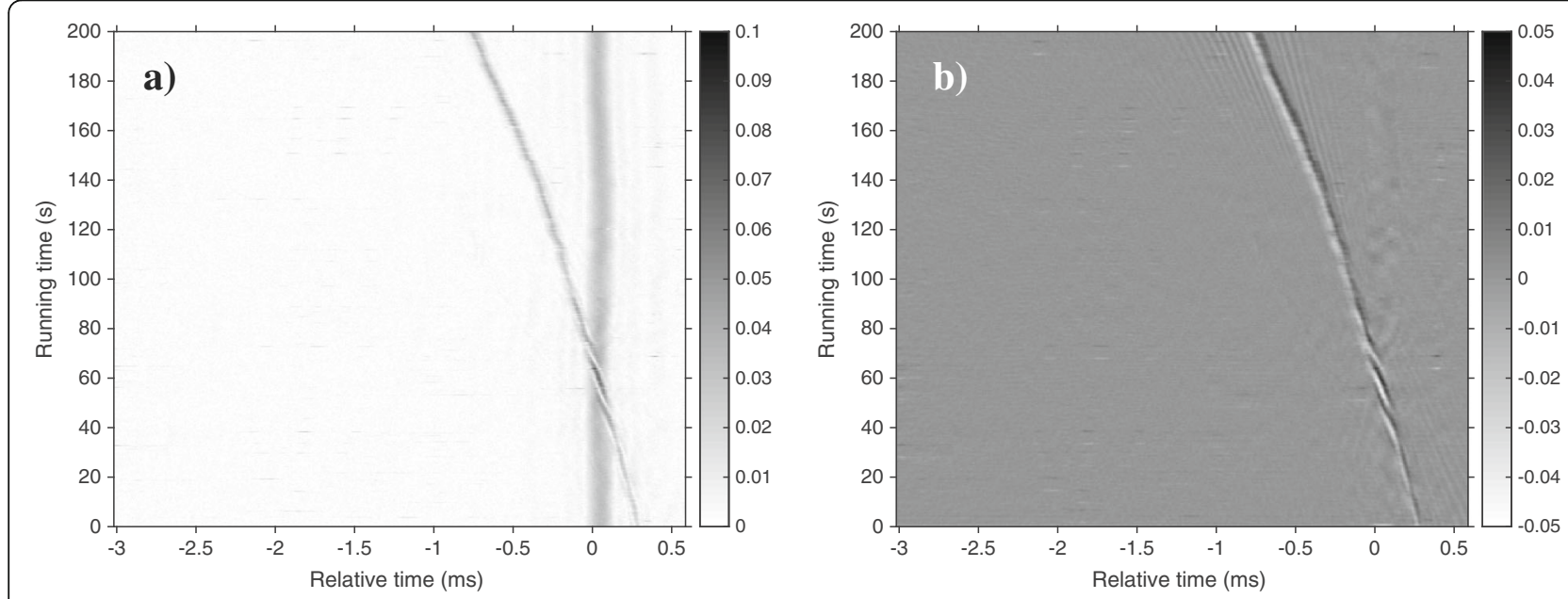

Fig. 6 Processing results for data received on the first hydrophone pair at a running time from 40 to 60 s, showing that the TDOAs of the target signal component, before (a) and after (b) interference cancellation, are almost the same 

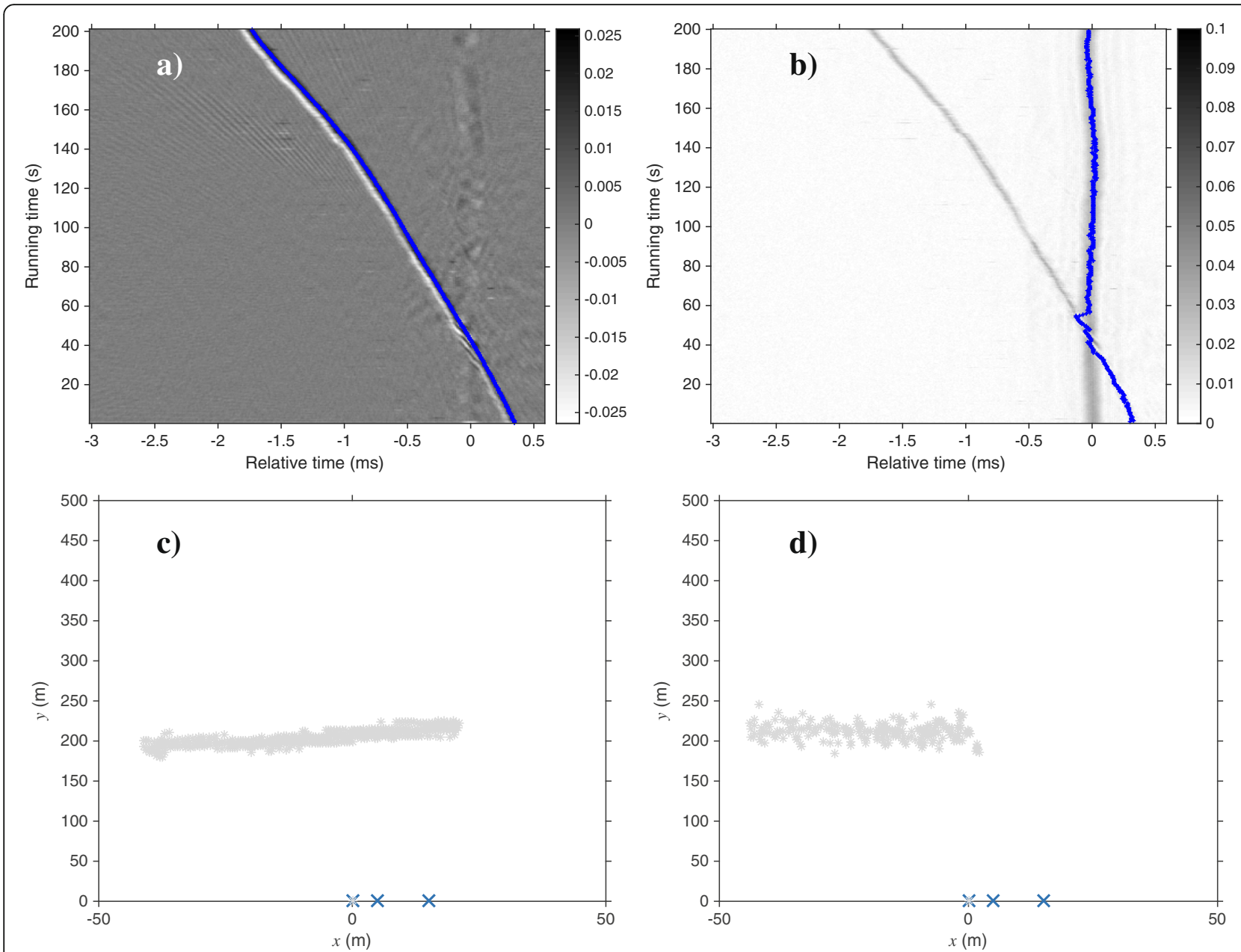

Fig. 7 TDOA estimation and localization results based on PHAT processing of the first pair of signals. a TDOA proposed method. b TDOA PF method (unavailable at the crossing event). $\mathbf{c}$ Localization proposed method. $\mathbf{d}$ Localization PF method (only the portion with the correct TDOAs is shown)

however, the jamming source may be moving as well as strong. Strong moving jamming sources could include a merchantman or military vessel. In this scenario, the same problem prevails in the TDOA estimation. Given that the trajectory does not exhibit a line on the PHAT outputs, additional preprocessing is required for TDOA estimation used in localization. When PHAT processing is performed, the strongest peaks from the outputs should be aligned and the deviations stored in memory. Subsequently, the interference cancellation method is performed by block processing. Afterward, the TDOA of the target signal is connected using the recorded deviations.

As an example, two moving sources are present in this experiment: one is the same boat whose trajectory is known based on its GPS, and the other is an unknown boat moving at a high speed. In processing, the second boat is considered the jamming source. The recorded waveforms are prefiltered the same as in Section 3 and processed in 0.5-s blocks. The two correlation outputs are displayed in Fig. 8a, corresponding to the jamming source (dark curve) and target source (light curve). The relative delays of the jamming source indicate that it moves in the opposite direction of the target source. At a running time of approximately $68 \mathrm{~s}$, the two sources are at the same position and thus have the same TDOA. Given the relative time delays of the interference alignment, interference cancellation is performed on the PHAT output, yielding the result shown in Fig. 8b. The strong moving jamming source is eliminated, whereas the target source is retained. Some portions of the interference are not well isolated because of variations in the jamming source when it moves, as mentioned in Section 2.2.

The relative time delays of the target source on the receiver pairs can then be obtained directly, even at a running time close to the crossing event. Finally, the localization results are obtained, as shown in Fig. 8c, which agrees well with the GPS measurements in Fig. 8d.

The Radon transform works well for line detection. When the signal-to-interference ratio is very weak, a weak variation in the PHAT output exists at the crossing 

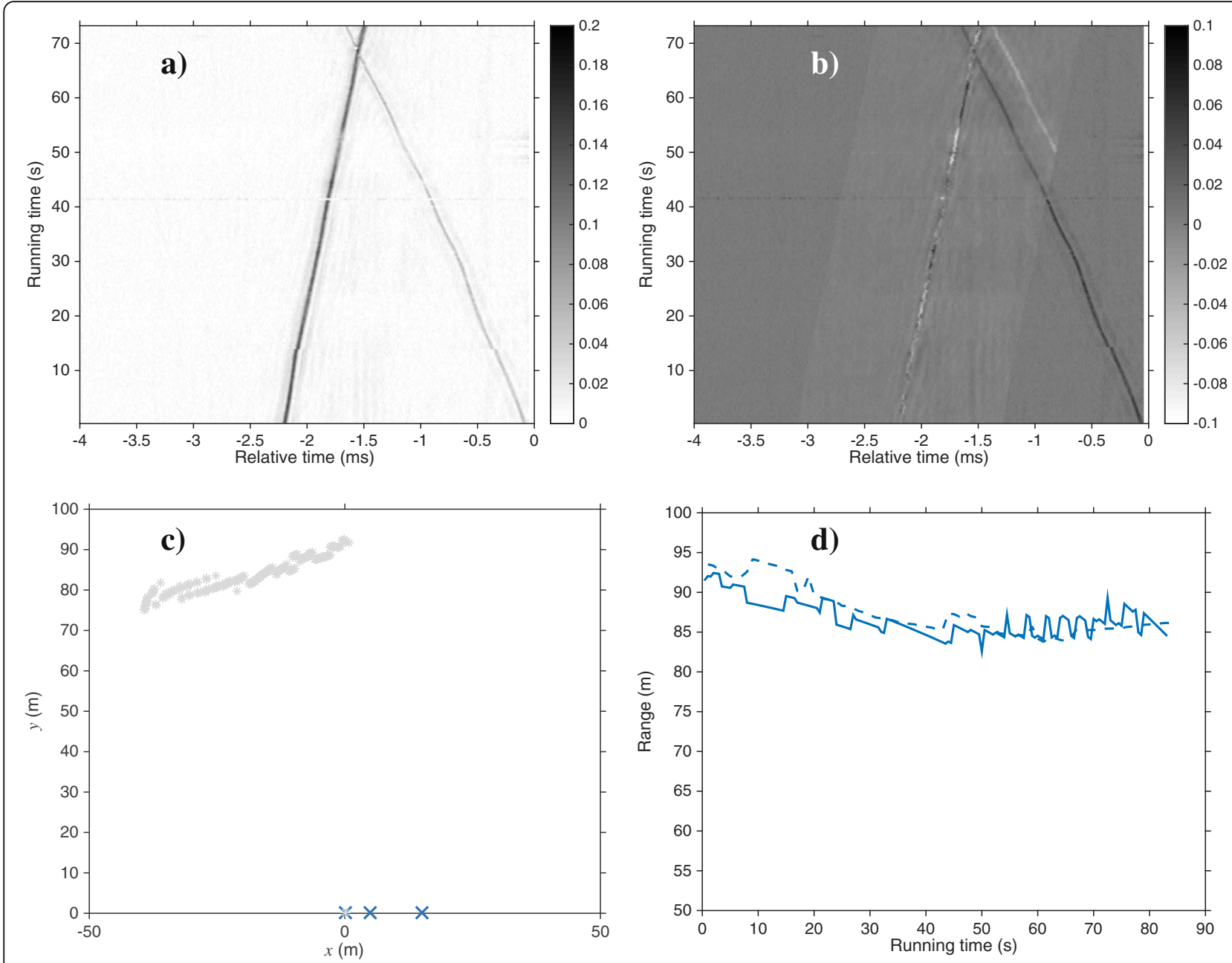

Fig. 8 Moving jamming source suppression. a PHAT outputs for the first pair in 0.5-s blocks. b Processing results with the strong jamming source subtracted. c Localization results. $\mathbf{d}$ GPS measurements (solid line and dashed line represent the evaluated and measured ranges, respectively)

event, such that the target signal will be eliminated as well during the interference cancellation. Consequently, the trajectory of the target signal will be interrupted at the crossing event, causing a gap in the estimated time delays. Given that the wideband jamming source (moving boat noise) has a good correlation function, the interference has only a slight influence on the TDOA estimation in the experiment. Nevertheless, this influence is sufficient to show the efficacy of the proposed method. If the jamming signal does not have a sharp correlation peak, this method may be more applicable.

\section{Conclusions}

In passive localization, the target signal is significantly contaminated by strong interference. As a result, traditional localization methods may be ineffective. In this study, a hybrid method involving PHAT processing, interference cancellation, and position searching is proposed. By certain additional preprocessing of the PHAT outputs, the interference can be adequately suppressed, allowing for good localization results in the preliminary experiments.

Although the experimental range is not the main concern of this study, the localization method can also achieve good performance at farther distances. A large system aperture is expected in that case, such as a long-baseline sensor array to achieve better localization. Furthermore, joint estimation is suggested for multiple localization systems when the number of receivers is more than three.

One possible application of this method is the monitoring of multiple moving acoustic sources with fixed hydrophones. A factor that is likely to impact the performance of this method is strong variation in the interference. This problem may be solved by applying a constant strength to the PHAT output setting over an appropriate threshold. In the experimental investigation, only direct arrival signals are considered. However, multipath propagation may not be negligible at longer 
ranges. Thus, a ray model may be necessary for time-delay estimation. A possible method to address this issue is to replace the analytical partial derivatives by a numerical method; however, this may require substantial computational resources.

\section{Acknowledgements}

The authors gratefully acknowledge the support for this research by the National Natural Science Foundation of China (61571366) and Natural Science Basic Research Plan in Shaanxi Province of China (2015JQ5199).

\section{Competing interests}

The authors declare that they have no competing interests.

Received: 23 June 2016 Accepted: 25 November 2016

Published online: 03 December 2016

\section{References}

1. JBY Tsui, Fundamentals of global positioning system receivers. (Wiley-Interscience, New York, 2000). doi:10.1002/0471200549

2. WK Ma, BN Vo, SS Singh, A Baddeley, Tracking an unknown time-varying number of speakers using TDOA measurements: a random finite set approach. IEEE Trans. Signal Process. 54(9), 3291-3304 (2006). doi:10.1109/TSP.2006.877658

3. NH Lehmann, AM Haimovich, RS Blum, L Cimini, Proc. Fortieth Asilomar Conference on Signals, Systems and Computers. High resolution capabilities of MIMO radar, 2006. doi:10.1109/ACSSC.2006.356576

4. M Bruno, KW Chung, H Salloum, A Sedunov, N Sedunov, A Sutin, H Graber, P Mallas, in Waterside Security Conference (WSS), 2010. doi:10.1109/WSSC. 2010.5730229. Concurrent use of satellite imaging and passive acoustics for maritime domain awareness

5. J Gebbie, M Siderius, R McCargar, JS Allen III, G Pusey, Localization of a noisy broadband surface target using time differences of multipath arrivals. J. Acoust. Soc. Am. 134(1), EL77-EL83 (2013). doi:10.1121/1.4809771

6. CH Knapp, GC Carter, The generalized correlation method for estimation of time delay. IEEE Trans. Acoust. Speech Signal Process. 24(4), 320-327 (1976). doi:10.1109/TASSP.1976.1162830

7. B Qin, H Zhang, Q Fu, Y Yan, Proc. 9th Int. Conf. on Signal Processing. Subsample time delay estimation via improved GCC PHAT algorithm, 2008. doi:10.1109/ICOSP.2008.4697676

8. MS Brandstein, HF Silverman, Proc. IEEE Int. Conf. Acoust. Speech, Signal Process. A robust method for speech signal time-delay estimation inreverberant rooms, 1997. doi:10.1109/ICASSP.1997.599651

9. J Chen, J Benesty, Y Huang, Time delay estimation in room acoustic environments: an overview. EURASIP J. Appl. Signal Processing. 170, (2006) doi:10.1155/ASP/2006/26503

10. DJ Torrieri, in Autonomous robot vehicles, ed. by IJ Cox, GT Wilfong (Springer, New York, 1990), pp. 151-166

11. YT Chan, KC Ho, A simple and efficient estimator for hyperbolic location. IEEE Trans. Signal Process. 42(8), 1905-1915 (1994). doi:10.1109/78.301830

12. DP Young, CM Keller, DW Bliss, KW Forsythe, Proc. 37th Asilomar Conf. Signals, Syst. Comput. Ultra-wideband (UWB) transmitter location using time difference of arrival (TDOA) techniques, 2003. doi:10.1109/ACSSC.2003.1292184

13. KW Lui, FKW Chan, HC So, Accurate time delay estimation based passive localization. Signal Processing 89(9), 1835-1838 (2009). doi:10.1016/j. sigpro.2009.03.009

14. B Friedlander, Accuracy of source localization using multipath delays. IEEE Trans. Aerosp. Electron. Syst. 24(4), 346-359 (1988). doi:10.1109/7.7176

15. P Felisberto, O Rodriguez, P Santos, E Ey, SM Jesus, Experimental results of underwater cooperative source localization using a single acoustic vector sensor. Sensors (Basel) 13(7), 8856-8878 (2013). doi:10.3390/s130708856

16. NY Ko, TG Kim, YS Moon, Proc. OCEANS Int. Conf. Particle filter approach for localization of an underwater robot using time difference of arrival, 2012. doi:10.1109/OCEANS-Yeosu.2012.6263573

17. J Gebbie, M Siderius, JS Allen III, A two-hydrophone range and bearing localization algorithm with performance analysis. J. Acoust. Soc. Am. 137(3), 1586-1597 (2015). doi:10.1121/1.4906835

18. F Antonacci, D Riva, D Saiu, A Sarti, M Tagliasacchi, S Tubaro, Proc. 14th European Signal Processing Conference, Tracking multiple acoustic sources using particle filtering, 2006
19. HM Shertukde, Y Bar-Shalom, Detection and estimation for multiple targets with two omnidirectional sensors in the presence of false measurements. IEEE Trans. Acoust. Speech Signal Process. 38(5), 749-763 (1990). doi:10.1109/29.56019

20. H Gauvrit, JP Le Cadre, C Jauffret, A formulation of multitarget tracking as an incomplete data problem. IEEE Trans. Aerosp. Electron. Syst. 33(4), 1242-1257 (1997). doi:10.1109/7.625121

21. A.P. Dempster, N.M. Laird, D.B. Rubin, Maximum likelihood from incomplete data via the EM algorithm. J. R. Stat. Soc. Ser. B. 39(1), 1-38 (1977).

22. D Carevic, Automatic estimation of multiple target positions and velocities using passive TDOA measurements of transients. IEEE Trans. Signal Process. 55(2), 424-436 (2007). doi:10.1109/TSP.2006.885745

23. SR Deans, The Radon transform and some of its applications (Dover Publication, New York, 2007)

24. G Beylkin, Discrete radon transform. IEEE Trans. Acoust. Speech Signal Process. 35(2), 162-172 (1987). doi:10.1109/TASSP.1987.1165108

25. MB Porter, Gaussian beam tracing for computing ocean acoustic fields. J. Acoust. Soc. Am. 82(4), 1349 (1987). doi:10.1121/1.395269

26. M.B. Porter, The BELLHOP manual and user's guide: PRELIMINARY DRAFT. http://oalib.hlsresearch.com/Rays/HLS-2010-1.pdf. Accessed 1 Mar 2011.

\section{Submit your manuscript to a SpringerOpen ${ }^{\circ}$ journal and benefit from:}

- Convenient online submission

- Rigorous peer review

- Immediate publication on acceptance

- Open access: articles freely available online

- High visibility within the field

- Retaining the copyright to your article

Submit your next manuscript at $>$ springeropen.com 